\title{
On Artistic Conception Beauty of Modern Lettering in Colleges and Universities
}

\author{
Guicai FU ${ }^{a^{*}}$, Wenjie $M^{b}$ and Pengfei FU ${ }^{c}$ \\ Department of fine arts and design, Heihe University, Heihe City, Heilongjiang, China \\ a435415992@qq.com, bliyanmin8888@sohu.com, 'liyanmin8888@sohu.com
}

Keywords: modern lettering, art artistic, conception beauty

\begin{abstract}
Artistic conception is a common high-level artistic realm in Chinese art, and it is an important form of expression for artists to express their emotions. The pursuit of artistic expression is an important part of traditional Chinese aesthetics. Through the analysis of the beauty of poetry, book and painting, this paper integrates the aesthetic thought of artistic conception into modern lettering, so as to improve the artistic taste of the works.
\end{abstract}

\section{Introduction}

The so-called "artistic conception" is to go beyond the concrete and limited physical state of the world, into the infinite time and space, so as to obtain a philosophical feeling and understanding of the whole life, history and the universe. The concept of artistic conception can be found in the theory of poetry. "poetry has three environments: one is the physical environment, the other is the situation, and the third is the artistic conception", which was first put forward by wang changling in his poem "shige"[1].The "artistic conception" in the theory of Chinese painting and calligraphy is the main body of artistic creation, through the description of the expression form of artistic works, and the revelation of its own spiritual and emotional factors, which gives the appreciators influence and blend[2].

The emergence of modern lettering art is no more than 30 years old. With its vigorous and vigorous youth, it soon occupies a place in China's broad art hall.It draws nutrition from the previous art categories and extracts the essence for its own use. In the limited creation layout, it displays the beauty of rich artistic conception through pen and ink, vertical and horizontal texture and myriad colors [3]

\section{Poetic Beauty of Modern Lettering Art}

In modern lettering, the first problem we encounter is to choose the content of lettering, and the determination of the content directly determines the level of the work. In the choice of words and sentences, must be considered deeply, in a limited physical space, in a limited range of words, try to express the artist to express the artistic information that the artist wants to express. Such content can provide infinite imagination space for appreciating the object, and even the appeal of art goes beyond the limit of the picture to force the emotional space of the creator, so that the creator and the viewer can communicate with each other in mind and get artistic feelings in harmony.

The selection of the content of a lettering work is also the establishment of the theme. It should have poetic language and artistic conception. It is the expression of the feelings of the creators and the result of the collision and integration of the subject and object. The formation of artistic conception is the external expression of the creator's thoughts and feelings after the deepening of the understanding of objective things. Ma zhiyuan's "tian jing sha qiu si" "withered vine old tree faint crow, small bridge water family......", although short, but by many people know, can be regarded as the last song. A short twenty eight characters, carved out a very real and vivid autumn sunset picture, a series of nine pictures: withered vine, old tree, faint crow, bridge, water, family, 
ancient road, west wind, thin horse, to the scene, scene of love, in the bleak background sketched out the traveler wandering and sad feelings. Here, the author creatively combines the isolated natural objects exquisitely to make the whole picture full of a sense of flow and life. At the same time, consciously highlight the dark and cold picture, in order to fully show the "heartbroken man" wandering around the world of strong fetters. This scene floating in front of us, the situation with the scene, make our creative impulse surging hard to stop. Such a picture also for our color, texture of the sculpture lay a foreshadow [4]. Another example is "distant mountain take sunset" in front of us is a picture of the western sunset, boundless mountains if rise if fall, green fade, leaving only in the sunset outline silhouette. Such a scene, such a theme, must be able to make the eyes of the viewer and the heart of the aesthetic feelings of your capture [5].

It is not hard to find through several national lettering art exhibitions , some authors' choice of lettering content is not deep and profound, lacking connotation, as if a piece of thin paper, a little effort can be understood. Its knife method, texture, layout, color may be very good, the use of letter-carving language is also appropriate, but to the audience feel is a lack of thickness, like a not full bow, lack of tension, lack of confidence. Simply focused on above works, in the form of sense, even for the sake of seeking pure forms of aesthetic feeling, for single piece of Chinese characters, the modelling of good Chinese characters are combined, or Chinese character component radical alternative far-fetched, such work has significant limitations, such work will be missing a high level of artistic conception of Chinese art beauty, is unsatisfactory.

Read more books, like to read tang poetry and song ci, and more with the ancients, over time will be able to milk in the predecessors' artistic realm, will also be able to pull out with a strong accumulation of character carving creation content, theme.

\section{The Beauty of Modern Lettering Art}

The real connotation of modern lettering is calligraphy, and what should be carved is the art of high level calligraphy. Since the object of expression is the art of calligraphy, we should use the rules of calligraphy and the artistic language of calligraphy in writing and designing samples.

From the font, true, grass, li, zhuan, line can be into the version, line, to stress the length, curve, thickness, back, even the line's dry run dry wet can be expressed in the lettering. In terms of rules and methods, they are both calligraphy language and elements of artistic conception of calligraphy. Only by making full use of the artistic language of calligraphy and thoroughly realizing the appeal of the artistic conception of calligraphy can we create excellent calligraphic works. Let the viewer not only appreciate the creator's skilled knife, exquisite color, but also appreciate the concentration of the excellent calligraphy works.

In modern lettering, the artistic conception of calligraphy cannot be isolated and unique. Calligraphy is important in modern lettering, but modern lettering is not only the stage of expression of calligraphy. Its beauty, its artistic conception, is constructed by many factors. To make it the subject of the content service, subject to the control of the content, coordinated in the entire layout. Such as our subject content of artistic conception is a quiet, long and long, graceful, magnificent, tender, delicate, such as always liuyiong's "willow bank morning winds waning moon" artistic conception, we choose the period and on the use of line will be in style, matching, or euphemistic, meaningful or elegance, or exquisite and delicate. For example, our theme content artistic conception is vast, rough, thick, wild, plain, such as su shi's "big river east, wave wash away" as artistic conception, in the choice of style and lines should be or solid, or bold, or sad, or powerful heroic. In a word, the artistic conception of calligraphy is related to the artistic conception of the whole lettering work, must not ignore the theme of the content of the style characteristics, free in the overall layout of artistic conception [6]. 


\section{The Beauty of Modern Lettering Art}

Modern lettering under the guidance of theme artistic conception should be consciously around the theme of the article. In the layout design, from the picture environment to absorb nutrients, extract the essence. In the layout to pursue "thin and dense, solid and virtual"; In artistic thought, we should pursue the blending of emotion and image. "Emotion" is the aesthetic emotion of the creative subject, while "image" is the psychological image of the subject's emotion. Modern lettering art is an art form based on the shape of Chinese characters. Through its unique re-creation of calligraphy art, it adopts such unique lettering language as exaggeration, superposition and substitution of Chinese strokes to move from two-enclosed space to three-enclosed and three-dimensional work form. This is the difference between modern lettering and other related arts, as well as the distinctive feature of traditional lettering. In modern lettering, the expression of mechanism is not to be ignored. The mark of chisel and the principle of carving can show different theme artistic conception. The macho and bold theme is supplemented by the decisive and courageous mechanism; The soft and delicate theme is designed to reveal the different emotional experience and emotional state of the creative subject [7].

Color plays an important role in the creation of artistic conception of modern lettering. It is the embodiment of the theme artistic conception. The proper use of color is helpful to the expression of the theme, and it is the standard to judge the quality of a work and the cultivation of the author. People who have studied color composition know that color also has color emotion, which is perceived by everyone with normal color sense. For example, red expresses warm emotion, green expresses vibrant emotion, and blue expresses dreamy emotion. This is the emotion conveyed by color, which is the emotional experience that we can make full use of on the picture.

In the design of the layout to "pen first", "with confidence". However, the brewing image is not very clear, but in the concrete operation of lettering art creation with the completion of the form gradually appeared, but also with the work process and gradually improved. When the original conception is satisfied, the artistic conception is expressed in the perfect unity of "image" and "meaning"[8].

Modern lettering art is not only a form of expression, but also a process of creating artistic conception. In order to perfectly convey the feelings and thoughts of the creator, the creative subject draws on the language symbols of various artistic categories and skillfully uses the unique artistic elements of lettering. All these are for one goal -- the creation of artistic conception in modern lettering art works. The beauty of artistic conception is the high level and eternal spiritual pursuit of modern lettering art.

\section{Project Fund}

This paper is the research result of the higher education teaching reform project of Heilongjiang Province in 2017, "research on teaching methods of modern lettering techniques". The project number is SJGY0212

\section{References}

[1] Hui Ji.On the source and flow of Chinese modern lettering art.[D].Qufu normal university,2018.

[2]Zongchao Liu.Modern lettering should return to the standard of calligraphy [N]. Art report,2017-12-02(017).

[3] Shenghong Li.Chinese modern lettering art:Is entering a new stage of historical development [N].Chinese calligraphy news,2019-11-05(001).

[4] Xuewei Mei On the beauty of artistic conception of modern lettering art [J]. Fujian forum (humanities and social sciences edition),2012(S1):9-10. 
[5] Mengqiang Jia.A study of the art of book carving [D]. Bohai university,2015.

[6] Xiuqing Chen.Reflections on the art of lettering [J]. Chinese calligraphy,2015(17):46-47.

[7] Huaping Song.Spread out the magnificent scroll of the new age lettering art [N]. Chinese calligraphy news,2019-11-12(004).

[8] Hongyi Liu.Exploration and practice of lettering techniques [J]. Border area economy and culture,2012(04):137-138. 